\title{
VORWORT
}

\section{ZUM BETRACHTUNGSZIEL DEUTSCHE SPRACHMINDERHEITEN IM ÖSTLICHEN EUROPA - SPRACHE, GESCHICHTE, KULTUR}

Die Idee, sich mit den deutschen Sprachminderheiten im östlichen Europa zu beschäftigen, reifte lange und auf verschiedenen Wegen. Den Entschluss der Herausgeberin und des Herausgebers, im wissenschaftlichen Diskurs schlussendlich darüber diskutieren zu wollen, haben jedenfalls einige ganz konkrete Momente geprägt: Zunächst waren das persönliche Begegnungen mit einigen wenigen Sprecherinnen und Sprechern des Gottscheerischen, die in einigen Gottscheer Dörfern (weiterhin) leben, und deren sprachbiographische Erzählungen uns für die in der Gegenwart verschwindende Sprache einnahmen; zum zweiten die Besichtigung einer aussagekräftigen Ausstellung über die Gottscheer im Regionalmuseum in Kočevje (Pokrajinski muzej Kočevje), deren Projektverantwortlicher Prof. Dr. Mitja Ferenc die Geschichte dieser Sprachinsel aus historiographischer Perspektive ausgezeichnet medialisierte; ein weiterer wichtiger Moment war ein in mehreren Gesprächen bekundetes Interesse des Regensburger Germanisten Prof. Dr. Hermann Scheuringer, des Leiters des Forschungszentrums Deutsch in Mittel-, Ost- und Südosteuropa (FZ DiMOS) der Universität Regensburg, an einer überregionalen wissenschaftlichen Thematisierung der Problematik der deutschen Sprachminderheiten in Slowenien.

Diese Fakten, Einsichten, Interessen und vor allem auch die Recherche des Forschungsstandes, besonders des germanistischen, zeigten, dass einige deutschsprachige Minderheiten, darunter beispielsweise auch die in der Gottschee oder in Marburg an der Drau/Maribor, einer intensiveren Einbettung in den - zwar regen - überregionalen europäischen wissenschaftlichen Diskurs über deutsche Sprachminderheiten im östlichen Europa bedürfen. Die Zusammenarbeit der Universität Ljubljana, der Universität Maribor und der Universität Regensburg durch die Herausgeberin und den Herausgeber und Prof. Scheuringer resultierte in der vorliegenden Publikation, in der weitere Forscherinnen und Forscher aus den Bereichen der Sprachwissenschaft, Geschichte, Literaturwissenschaft und Minderheitenforschung über ihre aktuellen Forschungen und Erkenntnisse zu Sprachen, Sprachkontakten, Literaturen, Geschichte und Kulturen der Sprachminderheiten berichten. Daher wird diese Publikation mit dem Thema „Sprachminderheiten und ihre Sprachen“ eröffnet.

Der einleitende Beitrag setzt sich mit traditionell verankerten Begriffen wie etwa östlich oder Sprachminderheit sowie mit aktuellen Gegebenheiten der deutschen Minderheiten im slowenischen Raum (Uršula Krevs Birk) auseinander. Weitere Beiträge zur Sprache betrachten diverse östliche Regionen Europas, wie etwa Oberschlesien und dortige Sprachkontaktphänomene der bilingualen Sprecher (Felicja Księżyk), ferner auch den schlesisch-kleinpolnischen Grenzraum und seine Sprachinsel Wilmesau (Grzegorz Chromik). Weitere Studien befassen sich mit Ungarn und den Auswirkungen 
der deutschen Dialekte auf bilinguale Sprecherinnen und Sprecher in deutschsprachigen Landesteilen Ungarns (Csaba Földes) sowie mit weiteren aktuellen sprachbiographischen Fragestellungen zu den Perspektiven der Minderheit in Ungarn (Elisabeth Knipf-Komlósi/Marta Müller) oder dem Stand des dortigen Minderheitenschulwesens (Koloman Brenner). Das Interesse der Forschung gilt auch der geschichtlichen Relevanz der deutschen Sprache in Siebenbürgen und ihrer Weiterentwicklung als Standardvarietät (Doris Sava), den deutschen Kolonien in Bosnien und Herzegowina (Sanja Radanović) sowie der deutsch-bosnischen Kulturvermittlung (Nedad Memić). Thematisiert werden auch relevante literatur- und kulturwissenschaftliche Inhalte wie etwa die Vermittlung der Minderheitenliteratur im zusammenhängenden deutschen Sprachraum (Sigurd Paul Scheichl), die Rolle des deutschen Theaters in Kroatien (Marijan Bobinac), das Schicksal der traditionsreichen deutschen Minderheitentheater in der Zwischenkriegszeit (Anselm Heinrich) und die Perspektiven weiterer Forschungen, beispielsweise zu den Wolgadeutschen (Egor Lykov).

In allen thematischen Teilen der vorliegenden Publikation (Sprache, Geschichte, Kultur) werden auch verschiedene Aspekte der deutschsprachigen Minderheiten im slowenischen Raum fokussiert: die Geschichte der Deutschen und der Gottscheer Sprachinsel (Mitja Ferenc) sowie deren historische und politische Wendepunkte (Ana Begovac), aber auch das Sagengut der Gottscheer unter dem Aspekt des Raumes (Matjaž Birk). Darüber hinaus geht es um die Rolle der Deutschen und der deutschen Sprache in Maribor/Marburg a. d. Drau aus sprachwissenschaftlicher (Vida Jesenšek) und historiographischer Sicht (Gregor Jenuš), um den Sprachenwechsel in der ehemaligen Sprachinsel Zarz/Sorica (Heinz-Dieter Pohl) wie auch um die deutsch-slowenischen Sprachkontaktprozesse anhand des Oberkrainer Dialektes aus Kropa (Uršula Krevs Birk/Domen Krištofelc) sowie die phonetischen Charakteristika der deutschen Entlehnungen aus einzelnen hochdeutschen Chronolekten (Matej Šekli).

Im vorletzten Abschnitt erscheinen zwei Beiträge, die „westlich“ von den zuvor untersuchten Kulturräumen anzusiedeln sind. Das Motiv für den Einbezug der Perspektive auf ,westliche“ Sprachminderheiten liegt nicht nur in der intendierten Beleuchtung der Minderheitenproblematik durch die Positionierung des Beobachters auf der mehrfach kodierten westlich-östlichen Achse, sondern schlicht auch in Vergleichen, die sich durch die Einsicht in die soziolinguistische Geschichte des Elsass (Thomas Nicklas) und der deutschsprachigen Minderheit in den USA (Tristan Coignard) ableiten lassen. Das abschließende Kapitel bringt zwei Buchbesprechungen, die wiederholt die Relevanz der Minderheitenforschung zum Ausdruck bringen.

Am Rande seien zwei Bemerkungen bezüglich der Toponymika und gendergerechten Sprache formuliert: Zunächst möchten wir als Herausgeber die in der Publikation verwendeten Toponymika herausstellen, deren Ein-, Zwei- oder Mehrsprachigkeit, Endonymie und Exonymie, Historizität und Aktualität zweifellos weitere Problem- und Diskussionsfelder eröffnen. Obwohl unsererseits eine einheitliche Vorgehensweise bei der Angabe der Institution verfolgt wurde, ist auf jedwede Vereinheitlichung bei der Wahl und Verwendung einzelner Toponyme in den Beiträgen verzichtet worden, da uns der Namengebrauch der Verfasserinnen und Verfasser bei der jeweiligen Themendarstellung 
als relevant erscheint. Die zweite Bemerkung betrifft die gendergerechte Sprache und das Dilemma - für den Hinweis darauf sei Prof. Dr. İnci Dirim gedankt -, inwiefern die Bezeichnungen in den Beiträgen, die von aus verschiedenen wissenschaftlichen Diskurstraditionen stammenden Autorinnen und Autoren verfasst wurden, zu vereinheitlichen sind. Nach langen Überlegungen ist die Entscheidung gefallen, den Sprachgebrauch der Verfasserinnen und Verfasser von Beiträgen zu respektieren, was möglicherweise auch als ein Reflex der auslandsgermanistischen Verortung zu verstehen ist.

Die Aufsätze von 24 Autorinnen und Autoren erscheinen nicht zufällig in der laufenden Nummer der Zeitschrift Linguistica, da dieses Wissenschaftsperiodikum in Slowenien an der Philosophischen Fakultät Ljubljana erscheint, zum Bedauern der Herausgeberin und des Herausgebers aber erst zum Jahresende 2020, was jedenfalls nicht nur, aber leider auch mit der unerwarteten weltweiten Ausbreitung der Coronapandemie zu tun hat, deren diverse Auswirkungen unsere Arbeit an der Publikation maßgeblich beeinträchtigten. Fürs Verständnis dafür sei allen Mitwirkenden daher ausdrücklich gedankt.

Die bereits am Anfang erwähnte Zusammenarbeit wurde in verschiedenen Phasen von mehreren Akteuren und Institutionen unterstützt, unter anderem von dem Regionalmuseum Kočevje (Pokrajinski muzej Kočevje), der Österreich-Bibliothek Marburg/ Maribor an der Universitätsbibliothek Maribor (Univerzitetna knjižnica Maribor), dem Deutschen Akademischen Austauschdienst (DAAD) und besonders auch von dem Forschungszentrum Deutsch im Mittel-, Ost- und Südosteuropa und seinen Mitarbeitern, wofür wir uns ausdrücklich bedanken. Für die finanzielle Unterstützung der Publikation gebührt der Dank dem Centre interdisciplinaire de recherche sur les Langues et la Pensée (CIRLEP) der Universität Reims Champagne-Ardenne und seinem Direktor, Prof. Dr. Thomas Nicklas, dem wir darüber hinaus auch für seine exemplarische kollegiale Begleitung der Vorbereitung der vorliegenden Publikation zu Dank verpflichtet sind. Für die finanzielle Förderung der Publikation geht unser Dank ebenfalls an das Österreichische Kulturforum Laibach und dessen Direktor, Herrn Andreas Pawlitschek.

$\mathrm{Zu}$ großem Dank verpflichtet sind wir aber Prof. Dr. Hermann Scheuringer, dem Leiter des Forschungszentrums DiMOS an der Universität Regensburg, der der Verwirklichung unseres Wissenschaftsvorhabens entscheidende Impulse verlieh.

Ljubljana, im November 2020

Uršula Krevs Birk und Matjaž Birk 